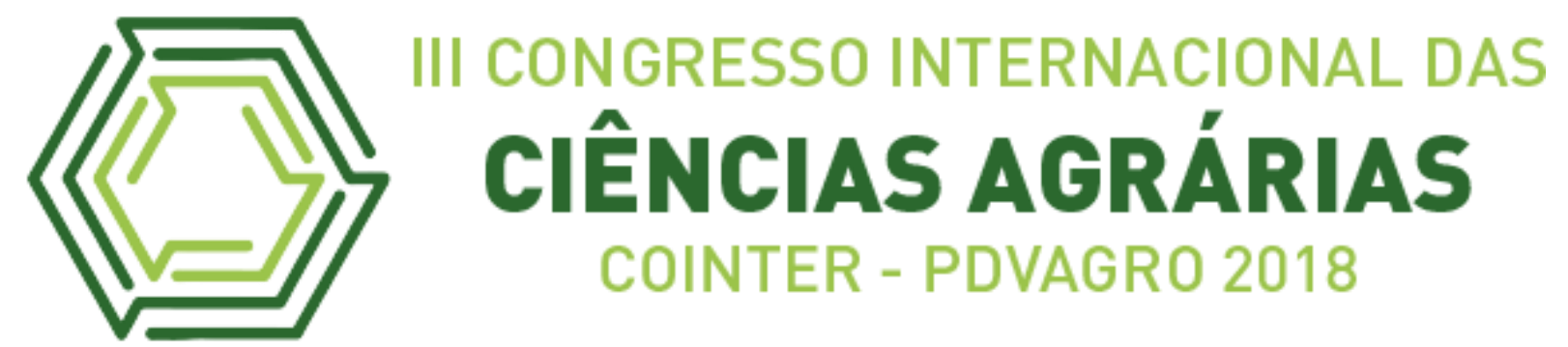

\title{
CARACTERÍSTICAS FÍSICO-QUÍMICAS DE RAÍZES DE MANDIOCA DE MESA
}

\section{PHYSICAL-CHEMICAL CHARACTERISTICS OF SWEET CASSAVA VARIETIES}

\author{
Apresentação: Pôster
}

Ramon Soares da Mata ${ }^{1}$; Fabrício Vieira Dutra ${ }^{2}$; Geovana Brito Queiroz ${ }^{3}$; Thais Soares Santana $^{4}$; Adriana Dias Cardoso ${ }^{5}$

\section{DOI: https://doi.org/10.31692/2526-7701.IIICOINTERPDVAGRO.2018.00352}

\section{Introdução}

A mandioca, Eudicotiledônea, da família euphorbiaceae, originária do Brasil, é cultivada mundialmente em cerca de 16 milhões de hectares (EL-SHARKAWY et al., 2008).

Essa cultura apresenta aceitação no mercado mundial, devido a suas raízes serem ricas em amido, que é fonte calórica muito importante, principalmente para famílias de baixa renda, além de ser considerada como um dos alimentos básicos das populações indígenas (COCKCROFT, 2004).

Apenas uma pequena parte é utilizada na alimentação animal ou como matéria-prima para produtos industrializados que não se destinam à alimentação humana. No Brasil, a mandioca integra o cardápio em todas as regiões brasileiras, desde o período pré-colonial até os dias atuais. É consumida na forma de farinha de mandioca ou de derivados de amido ou, ainda, as raízes são processadas no âmbito doméstico e consumidas cozidas, fritas ou utilizadas para preparar pratos típicos. Neste caso, são chamadas de mandioca de mesa ou de uso culinário (MEZETTE et al., 2009).

O consumo culinário de raízes de mandioca é bastante generalizado em todo o mundo, sendo amplamente utilizadas na forma cozida, assada e frita ou integrando pratos mais complexos. Atualmente, a mandioca para uso culinário é comercializada como vegetal fresco

\footnotetext{
${ }^{1}$ Graduando em Agronomia, Universidade Estadual do Sudoeste da Bahia - UESB, ramondamata@hotmail.com

2 Mestrando em Fitotecnia, Universidade Estadual do Sudoeste da Bahia - UESB, fabríciovieira94@hotmail.com

${ }^{3}$ Graduando em Agronomia, Universidade Estadual do Sudoeste da Bahia - UESB, geovanabritoq@hotmail.com

${ }^{4}$ Graduando em Agronomia, Universidade Estadual do Sudoeste da Bahia - UESB, thaissspfc@gmail.com

${ }^{5}$ Pesquisadora CAPES/PNPD, Universidade Estadual do Sudoeste da Bahia - UESB, adriuesb@yahoo.com.br.
} 
ou minimamente processada, refrigerada ou congelada, ou também na forma pré-cozida facilitando o preparo e consumo (OLIVEIRA et al., 2005).

A diferença mais concreta entre variedades para uso industrial e para mesa encontra-se no modo de consumo. As bravas são utilizadas para produzir farinha, extrair amido e outros produtos, mas somente são consumidas após algum tipo de processamento industrial com efeito destoxificante. Variedades mansas são mais versáteis, podem ser destinadas ao processamento tal qual as variedades bravas, e também consumidas após preparos mais simples como cozidas, fritas ou assadas (VALE et al., 2004). Diante disso, o objetivo deste trabalho foi avaliar características químicas e físicas de raízes de sete variedades de mandioca de mesa no município de Vitória da Conquista, Bahia.

\section{Fundamentação Teórica}

A cultura da mandioca está presente nas diversas regiões do mundo por apresentar tolerância às condições adversas de clima e solo. As raízes tuberosas são uma das mais importantes fontes de carboidratos e de subsistência para as populações mais carentes e as folhas são ricas em proteínas, vitaminas A e C, além de outros nutrientes (FUKUDA, 2005).

O teor de cianeto total encontrado nas variedades de mandioca determina a sua nomenclatura e o processo de industrialização e comercialização. Mandioca com menos de $100 \mathrm{mg}$ de $\mathrm{HCN} \mathrm{kg}^{-1}$, são denominadas mandioca mansa, doce ou de mesa, comumente chamada no Nordeste de macaxeira, destinada ao uso culinário ou de mesa. Mandioca com mais de $100 \mathrm{mg}$ de $\mathrm{HCN} \mathrm{kg}^{-1}$, são denominadas de mandioca brava ou amargas, destinadas ao processo industrial (SOUZA, 2009).

A comercialização das raízes de mandioca para uso na alimentação humana se dá principalmente sob a forma in natura, ou seja, do uso direto. Entretanto, no Brasil é crescente o mercado de produtos de mandioca de uso culinário industrializados como a pré-cozida congelada, os produtos processados a partir da massa cozida, como croquetes, empanados, bolinhos condimentados e massas formatadas, como os palitos estruturados e ainda as fritas tipo chips (FENIMAN, 2004).

A qualidade culinária de raízes frescas é um parâmetro importante na seleção de variedades de mandioca de mesa. A identificação dessa qualidade envolve fatores variados e complexos por constituir-se de um conjunto de características físicas, químicas e sensoriais, algumas das quais são determinadas objetivamente, como teores de cianeto, amido, fibra e 
tempo de cocção entre outras, subjetivamente, como sabor, consistência e firmeza da polpa cozida (BORGES et al., 2002).

\section{Metodologia}

Raízes tuberosas de sete variedades de mandioca de mesa (Cacau, BRS Milagrosa, Pão da China, Pretona, BRS Gema de ovo, Pacaré e Julieta) foram coletadas em propriedades rurais do município de Vitória da Conquista - Bahia e encaminhadas ao Laboratório de Melhoramento e Produção Vegetal, da Universidade Estadual do Sudoeste da Bahia, Campus de Vitória da Conquista - Bahia para avaliação química.

As características avaliadas foram: a) pH: em amostras de raízes in natura, utilizando-se pHmetro Marte, modelo MB-10; b) Acidez total titulável (\% de ácido cítrico.100g-1 de polpa): determinada por meio de titulação com solução de $\mathrm{NaOH} 0,1 \mathrm{~N}$ e indicador fenolftaleína, de acordo com o Instituto Adolfo Lutz (1985); c) sólidos solúveis ( ${ }^{\circ}$ BRIX) realizadas conforme o método da AOAC (ASSOCIATION OF OFFICIAL ANALYTICAL CHEMISTS, 1997); e cor da raiz crua: determinou-se com a utilização do Colorímetro Minolta, modelo Chroma Meter CR-200b. Foram avaliadas no sistema L, a* e b* (BIBLE e SINGHA, 1993). A variável L indica luminosidade, diferenciando cores claras de escuras. Seu valor varia de zero para cores escuras (preto) a 100 para cores claras (branco). O $\mathrm{a}^{*}$ indica intensidade das cores, variando do verde (-60) ao vermelho $(+60)$, e o b* indica intensidade das cores, variando de azul (-60) a amarelo (+60).

Os resultados foram analisados por análise de variância utilizando-se o software SAEG, versão 9.1 e os testes de médias pelo procedimento DUNCAN, em nível de 5\% de significância.

\section{Resultados e Discussões}

Todas as características avaliadas apresentaram diferença significativa entre as variedades (Tabela 1). Em relação ao pH, os valores variaram entre 6,65 e 6,32 tendo a variedade BRS Gema de ovo com o maior valor $(6,65)$. Resultados próximos aos encontrados por Teixeira et al. (2017), avaliando 20 variedades de mandioca de mesa, obteve variação entre 6,84 e 6,32, com pH de 6,69 para a variedade BRS Gema de ovo. A acidez constitui fator de grande importância para o sabor e aroma dos frutos, cujo $\mathrm{pH}$ influencia o escurecimento oxidativo dos tecidos vegetais (LEME et al., 2015). 
Tabela 1. pH, acidez titúlavel, solidos soluveis totais ( $\left.{ }^{\circ} \mathrm{BRIX}\right)$ e cor (L, a*, $\left.\mathrm{b}^{*}\right)$ de 7 variedades de mandioca de mesa. Vitória da Conquista, Bahia.2018. Fonte: Própia

\begin{tabular}{lllllll}
\hline VARIEDADES & $\mathrm{pH}$ & $\mathrm{AT}(\%)$ & $\mathrm{S}$ SST & \multicolumn{3}{c}{ COR } \\
\cline { 6 - 8 } & & & $\left({ }^{\circ} \mathrm{Brix}\right)$ & \multicolumn{1}{c}{$\mathrm{L}$} & $\mathrm{a}^{*}$ & $\mathrm{~b}^{*}$ \\
\hline CACAU & $6,48 \mathrm{BC}$ & $0,40 \mathrm{~A}$ & $6,97 \mathrm{C}$ & $91,54 \mathrm{~A}$ & $-0,70 \mathrm{BC}$ & $3,23 \mathrm{D}$ \\
BRS MILAGROSA & $6,35 \mathrm{DE}$ & $0,25 \mathrm{~B}$ & $6,90 \mathrm{C}$ & $90,98 \mathrm{AB}$ & $-1,25 \mathrm{D}$ & $3,58 \mathrm{BC}$ \\
PÃO DA CHINA & $6,41 \mathrm{CD}$ & $0,23 \mathrm{~B}$ & $6,57 \mathrm{D}$ & $88,90 \mathrm{BC}$ & $-0,93 \mathrm{CD}$ & $3,17 \mathrm{D}$ \\
PRETONA & $6,32 \mathrm{E}$ & $0,26 \mathrm{~B}$ & $6,90 \mathrm{C}$ & $89,66 \mathrm{ABC}$ & $-1,01 \mathrm{CD}$ & $3,38 \mathrm{CD}$ \\
BRS GEMA DE OVO & $6,65 \mathrm{~A}$ & $0,27 \mathrm{~B}$ & $7,33 \mathrm{~B}$ & $87,99 \mathrm{CD}$ & $-0,83 \mathrm{CD}$ & $5,22 \mathrm{~A}$ \\
PACARÉ & $6,34 \mathrm{DE}$ & $0,39 \mathrm{~A}$ & $7,97 \mathrm{~A}$ & $86,15 \mathrm{D}$ & $0,13 \mathrm{~A}$ & $5,18 \mathrm{~A}$ \\
JULIETA & $6,55 \mathrm{~B}$ & $0,20 \mathrm{~B}$ & $8,07 \mathrm{~A}$ & $88,06 \mathrm{CD}$ & $-0,26 \mathrm{AB}$ & $3,77 \mathrm{~B}$ \\
\hline CV $(\%)$ & 0,69 & 22,59 & 1,44 & 1,48 & $-40,66$ & 4,33 \\
\hline
\end{tabular}

Médias seguidas pela mesma letra, na coluna, não diferem entre si pelo testeDuncan, a 5\% de probabilidade.

As variedades que apresentaram maior acidez titúlavel foram Cacau e Pacaré com 0,40 e 0,39 respectivamente. Os parâmetros indicadores de sabor ácido ou azedo e também utilizados na determinação da qualidade dos produtos processados são a acidez titulável e o pH, Bezerra (2000).

A variedade Pacaré apresentou $7,97^{\circ}$ BRIX, junto com a Julieta com $8,07^{\circ}$ BRIX, as variedades com maiores valores. Os teores de acidez titulável foram inferiores aos encontrados por Carrerà et al. (2014), que foram de 2,3-3,6\%, porém a quantidade de SST ( ${ }^{\circ}$ BRIX) foi de 1,0-1,4 ${ }^{\circ}$ BRIX, inferiores aos obtidos neste trabalho, essa variação ocorre provavelmente, devido as diferentes variedades avaliadas nos estudos.

Para as avaliações de cor, as variedades Cacau, BRS Milagrosa e Pretona apresentaram o valor de L (luminosidade) mais próximos de 100, com 91,54, 90,98 e 89,66, portando tendo coloração da polpa tendendo ao branco. Para a intensidade das cores que varia entre verde e vermelho $(* a)$, os valores foram próximos, tendo a variedade Pacaré o único valor positivo com 0,13 , seguida pela Julieta com $-0,26$. Para a característica de intensidade das cores que varia entre azul e amarelo (*b), as variedades BRS Gema de ovo e Pacaré, foram as que apresentaram maiores números sendo a primeira com 5,22 e a segunda com 5,18, portando foram as que mais se aproximaram da cor amarela, essa coloração é uma característica marcante dessas variedades. Segundo Fialho et al. (2007), o mercado do Distrito Federal tem uma exigência por variedades de raízes de polpa creme ou amarela, entretanto, na região Norte e no Maranhão, a preferência é por raízes com polpa branca para mandioca de mesa e amarela para a produção de farinha (FUKUDA et al., 2006). Ponte (2008) afirma que, 
no município de Vitória da Conquista, a Pacaré e a Cacau Amarela, têm boa aceitação no mercado, embora a preferência do consumidor local seja por variedades que apresentem polpa de cor branca ou creme.

\section{Conclusão}

As variedades de mandioca estudadas apresentaram diferença entre as características avaliadas, tendo possibilidade de atender as exigências de diferentes mercados consumidores de raízes tuberosas de mandioca de mesa.

\section{Referências}

ASSOCIATION OF OFFICIAL ANALYTICAL CHEMISTS. Official methods of analysis of AOAC International. 16th ed., 3rd rev. 2 v. Gaithersburg, MD, 1997.

BEZERRA, V. S. Alterações na composição química e cocção de raízes de mandioca (Manihot esculenta Crantz) minimamente processadas. Dissertação Mestrado. Universidade Federal de Lavras. Lavras. MG. 2000.

BIBLE, B.B.; SINGHA, S. Canopy position infleneces CIELAB coordinates of peache color. HortScience, v. 28, n. 10, p. 992-993, 1993.

BORGES, F. M.; FUKUDA, W. M. G.; ROSSETTI, A. G.; Avaliação de variedades de mandioca para o consumo humano. Pesquisa Agropecuária Brasileira, Brasília, DF, v. 37, n. 11, p. 1559-1565, 2002.

CARRÉRA, A. G. P.; CUNHA, R. L.; CUNHA, E. F. M.; REGO, J. Y. N. Características físico-químicas de raízes de mandioca mansa (Manihot esculenta Crantz). $18^{\circ}$ Seminário de Iniciação Científica e $2^{\circ}$ Seminário de Pós-graduação da Embrapa Amazônia Oriental. 12 a 14 de agosto de 2014, Belém-PA.

COCKCROFT, L., Current and projected trends in African agriculture: implications for research strategy. In: Hughes, J. D'A.; Adu, B. O. eds. Plant Virology in Sub-Saharan Africa. Ibadan, Nigeria: IITA/Abidjan, Côte d'Ivoire: ORSTOM, 2004. p.88-172.

EL-SHARKAWY, M.A.; LOPEZ,Y.; BERNAL, L.M. Genotypic variations in activities of PEPC and correlations with leaf photosynthetic characteristics and crop productivity of cassava grown in low-land seasonally-dry tropics. Photosynthetica, v. 46, cap.2, p. 238-247, 2008 .

FENIMAN, C.M.; Caracterização de raízes de mandioca (manihot esculenta crantz) do cultivar iac 576-70 quanto a cocção, composição química e propriedades do amido em duas épocas de colheita. Dissertação mestrado. Universidade de São Paulo. PIRACICABA. SP. 2004. 
FIALHO, J.de F.; FUKUDA, W.M.G.; PEREIRA, A.V.; JUNQUEIRA, N.T.V.; GOMES, A.C. Avaliação de variedades de mandioca de mesa nas condições de cerrado do Distrito Federal. Planaltina, DF: Embrapa Cerrados. 2002. 20p. (Boletim de Pesquisa e Desenvolvimento $\mathrm{n}^{\circ} 73$ ).

FUKUDA, W. M. G. Embrapa pesquisa mandioca para indústrias de amido. Desenvolvimento da indústria de fécula de mandioca no Brasil tem demandado novas variedades com teores de amido mais elevados nas raízes e qualidade que agregue valores ao produto. Associação Brasileira dos Produtores de Mandioca. Revista eletrônica, ano III, $n^{\circ} 11$. jul/set. 2005.

FUKUDA, W. M. G.; FUKUDA, C.; DIAS, M. C.; XAVIER, J. J. B. N.; FIALHO, J. F. Variedades. In: Aspectos Socioeconômicos e Agronômicos da Mandioca. Editor: Luciano da Silva Souza ... [et al.]. - Cruz das Almas: Embrapa Mandioca e Fruticultura Tropical, 2006a. p.433-454.

INSTITUTO ADOLFO LUTZ - IAL. Métodos físico-químicos para análise de alimentos. ed. 4, São Paulo: Instituto Adolfo Lutz, p. 1020, 2008.

LEME, A.C.; GROPPO, V.D.; ROMERO, A de. C.; SPOTO, M.H.F.; JACOMINO, A.P. Influência do uso de películas comestíveis em laranja 'pêra' minimamente processada. B.CEPPA, Curitiba, v. 25, n. 1, p. 15-24, jan./jun. 2007.

MEZETTE, T. F.; CARVALHO, C. R. L.; MORGANO, M. A.; SILVA, M. G da.; PARRA, E. S. B.; GALERA, J. M. S. V.; VALLE, TERESA. LOSADA.; Seleção de clones-elite de mandioca de mesa visando a características agronômicas, tecnológicas e químicas. Bragantia, Campinas, v.68, n.3, p.601-609, 2009.

OLIVEIRA, M.A.; LEONEL, M.; CABELLO, C.; CEREDA, M.P.; JANES, D.A. Metodologia para avaliação do tempo de cozimento e características tecnológicas associadas em diferentes cultivares de mandioca. Ciência e Agrotecnologia, Lavras, v.29, n.1, p.126$133,2005$.

PONTE, C. M de. A. Épocas de colheita de variedades de mandioca. DISSERTAÇÃO MESTRADO. Universidade Estadual do Sudoeste da Bahia. Vitória da Conquista. BA. 2008.

SOUZA, A. V. Tratamento térmico na manutenção de lichias armazenadas sob refrigeração. 2009. 54 p. Dissertação (Mestrado Agronomia). Universidade Estadual Paulista, Faculdade de Ciências Agronômicas, Botucatu.

TEIXEIRA, P. R. G.; VIANA, A. E. S.; CARDOSO, A. D.; MOREIRA, G. L. P.; MATSUMOTO, S. N.; RAMOS, P. A. S. Physical-chemical characteristics of sweet cassava varieties. Revista Brasileira de Ciências Agrárias. Recife, v.12, n.2, p.158-165, 2017.

VALLE, T. L.; CARVALHO, C. R. L.; RAMOS, M. T. B.; MUHLEN, G. S.; VILLELA, O. V.; Conteúdo cianogênico em progênies de mandioca originadas do cruzamento de variedades mansas e bravas. Bragantia, Campinas, v.63, n.2, p.221-226, 2004. 